\title{
DETERIORA SEQUOR. INTERPRETACIÓN NEUROFILOSÓFICA DEL FENÓMENO DE LA AKRASÍA ${ }^{1}$
}

\author{
PEDRO JESÚS TERUEL \\ Universitat de València
}

\begin{abstract}
RESUMEN: La aproximación al fenómeno de la akrasía desde la interpretación filosófica de los datos neurocientíficos trae consigo rendimientos teóricos y prácticos. Entre los teóricos se encuentra la fundamentación neurofisiológica de las acciones acráticas, con el consiguiente aumento de capacidad explicativa respecto de las argumentaciones clásicas ligadas a la noción de hábito. Entre los prácticos se halla su proyección terapéutica en el campo de la psicología y la psiquiatría. Sobre esta base propongo dicha aproximación como teoría con valor explicativo y predictivo que conjuga la reducción interteórica lograda con la evitación del reduccionismo ontológico.
\end{abstract}

PALABRAS CLAVE: neurofilosofía, akrasía, hábito, potenciación a largo plazo, reduccionismo ontológico.

\section{Deteriora sequor. A neurophilosophical interpretation of the phenomenon of akrasia}

\begin{abstract}
Approaching the phenomenon of akrasia from the philosophical explanation of neuroscientific data delivers both theoretical and practical outputs. The neurophysiological foundation of the akratic actions belongs to the first ones, with the consequent increase of explicative capacity beyond the classic argumentation based on the notion of habit. Its therapeutic application to the fields of psychology and psychiatry belongs to the practical outputs. I propose therefore this approach as a theory with explicative and predictive value which achieves intertheoretical reduction and avoids ontological reductionism at the same time.
\end{abstract}

KEY WORDS: neurophilosophy, akrasia, habit, long-term potentiation, ontological reductionism.

Uno de los mayores desafíos con los que se confrontan filósofos y filósofas es la cuestión de la libertad. Su afirmación o negación —a menudo, como corolario de argumentos teóricos de corte metafísico o antimetafísico- atañe a la imagen que el ser humano forja de sí mismo en cada época e incide en la trama de las cosmovisiones que constituyen la forma mentis de una determinada sociedad o cultura. Durante las últimas décadas, las investigaciones que proceden del ámbito interdisciplinar de la neurociencia han aportado nuevos argumentos que reavivan antiguos debates.

1 Esta contribución se enmarca en los trabajos del proyecto de I+D «Racionalidad práctica en perspectiva neuroética», financiado por el Ministerio de Economía y Competitividad del Gobierno de España (FFI2013-47136-C2-1-P). Forma parte de un proyecto plurianual en torno al alcance y los límites del pensamiento naturalista. Agradezco de corazón las valiosas aportaciones que los y las integrantes del equipo de investigación llevaron a cabo en torno al presente estudio en la sesión celebrada el 16 de mayo de 2016 en la Facultad de Filosofía de la Universitat de València. 
Mi intención en las páginas que siguen consiste en abordar uno de los elementos que integran esos debates: el problema de la akrasía. Para ello me interesará tener en cuenta los datos aportados por la neurociencia. Esto implica, de entrada, abandonar el ámbito de la investigación fenomenológica pura: en efecto, para alcanzar nuestro objetivo resulta crucial afrontar la recepción del problema en una de las regiones ontológicas que posibilitan su tratamiento.

Puesto que la neurociencia constituye el resultado de una parcelación metodológica, su interpretación admite variantes que van desde el reduccionismo metodológico al reduccionismo ontológico. Con «reduccionismo metodológico» $[\mathrm{RM}]$ nos referimos a la estrategia que consiste en parcelar el objeto de estudio (en este caso, los procesos mentales) para abordarlo desde un concreto punto de vista (aquí, sus bases neurofisiológicas) sin olvidar que su resultado arroja una visión parcial del objeto. Con «reduccionismo ontológico» [RO] aludimos a la interpretación de los resultados de un RM según la cual se cifra en ellos el entendimiento exhaustivo del problema, como si el punto de vista coincidiese con la esfera ontológica del fenómeno. En la estrategia propia del RO distingo entre dos operaciones: la intrateórica y la interteórica. La historia de la ciencia ha sido escenario de interesantes y en ocasiones exitosos ejemplos de RO intrateórico; es el caso del galileano en el campo de la física, del darwiniano en biología o del freudiano en psicología. En cambio, el RO interteórico da lugar a monismos que resultan problemáticos por su carácter unilateral y simplista ${ }^{2}$.

Pues bien: dilucidar si la aproximación neurocientífica al problema de la libertad —en el preciso fenómeno que hemos seleccionado para esta investigación- se desplaza del RM al RO, y cuáles sean sus proyecciones en la esfera práctica, constituye el objetivo del presente paper.

\section{DELIMITACiÓN DEL PROBLEMA}

En la enorme variedad de fenómenos de deliberación racional se presenta un caso llamativo por su estructura, en cierto sentido, paradójica. Puede ser que la deliberación arroje un resultado unívoco (que una cierta opción resulte preferible a otras) y que, en cambio, la acción se oriente hacia una de las opciones que habían sido desechadas; más aún, que esto suceda sin fundamento racional, o con un fundamento que el propio sujeto estima insuficiente tanto durante la fase de deliberación como en el ínterin que transcurre entre la elección y la realización misma de la acción. La paradoja estriba en que, a pesar de la apariencia determinista del proceso - el sujeto se ve abocado a un acto que racionalmente no ha preferido-, el proceso de deliberación se ha dado de

2 Sobre la distinción entre RM, RO y RO intrateórico e interteórico me he extendido en otro lugar (Teruel, 2013c). 
hecho, de manera que el conjunto no puede ser excluido del ámbito moral de la toma de decisiones.

Lo que acabo de describir está emparentado con un fenómeno al que la ética clásica dedicó considerables esfuerzos. Se trata del problema de la akra$s i ́ a^{3}$. Ante dicho problema caben dos actitudes básicas. La primera consiste en la negación: puesto que nadie elige a sabiendas su propio mal, los fenómenos mentados han de incluir un error deliberativo; es la opción de Platón en el Protágoras. Recientemente Donald Davidson ha hecho suya esta tesis al afirmar que en los casos de akrasía aparente lo que en realidad se produce es un juicio moral sólo parcial, mientras que el juicio global de la situación pondría de manifiesto la incorrección del proceder deliberativo anterior.

La segunda opción reside en el reconocimiento del hecho. El presente texto parte de esta asunción. El lector que niegue su existencia puede considerar, por tanto, que las páginas que siguen carecen de valor, puesto que presuponen una interpretación errada de ciertas acciones humanas. Por mi parte, en cambio, considero innegable la existencia de los fenómenos acráticos; y esto, sobre la base de argumentos que proceden de la estructura esencial de experiencias humanas posibles y reales:

a) La mera posibilidad de elección sobre la base de deliberaciones racionales no excluye lógicamente que pueda elegirse a sabiendas una opción respecto de la cual otras resultan preferibles (aun conociendo, de hecho, la preferibilidad de éstas). En efecto, se trata de órdenes eidéticos diferentes: el del conocimiento y el de la acción. Concebirlos como instancias que se solapan sin fisuras implicaría negar la distancia que existe entre intelección y voluntad.

b) La experiencia humana presenta numerosos casos de elección contraria a la deliberación racional previa, como atestigua el fenómeno del arrepentimiento concomitante a la realización de la acción. Pensemos, por ejemplo, en un sujeto que decide vengarse de otro a pesar de reconocer que la venganza no es un camino racionalmente preferible a dejar que la justicia siga su curso; en el dolor que siente una persona que ama a su pareja y que sin embargo se siente arrastrada a una experiencia de infidelidad; o en el caso del fumador que sigue fumando a pesar de que es consciente de que sería mejor no hacerlo (más adelante retomaremos estos casos, que representan tres modelos distintos de acción acrática).

c) El fenómeno de la akrasía constituye, desde el punto de vista eidético, el reverso de la libertad. Constituye la garantía de que la posibilidad

3 Del verbo griego $\kappa \rho \alpha \tau \dot{\varepsilon} \omega$ («ser fuerte, tener fuerza o poder») precedido por una

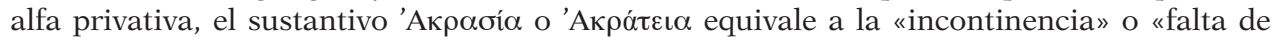
dominio de sí mismo». El texto fundamental al respecto en época clásica es el libro VII de la aristotélica Ética a Nicómaco (cf. en particular 1147b-1148b); distintos traductores optan por verter el término al castellano como «incontinencia» (es el caso de Julio Pallí: Ética nicomáquea, Gredos, Madrid 2011). Con todo, y dada la pluralidad de matices no pertinentes que adhieren al término castellano, prefiero emplear aquí la versión transliterada del griego. 
de elección es real, es decir, de que no equivale a la mera aplicación de un protocolo cuyo resultado se halla determinado de antemano (como sucede en el caso de la implementación de un programa informático). En efecto, supone que los órdenes del conocimiento y de la acción son mutuamente implicantes pero no mutuamente determinantes. Este argumento se halla emparentado con el primero y constituye su trasunto antropológico.

Sobre esta base, tomar en serio el problema de la akrasía me parece irrenunciable. Propongo aquí reflexionar sobre él a la luz de los datos que aporta la neurociencia. A mi modo de ver, la perspectiva neurocientífica nos permite afrontar con solvencia un fenómeno que la reflexión clásica consiguió enfocar con precisión relativamente menor.

\section{APROXIMACIÓN TEÓRICA AL FENÓMENO DE LA AKRASÍA}

Me ocuparé en primer lugar del modo en que una explicación más precisa de las bases neurofisiológicas de nuestra actividad mental puede contribuir a la comprensión filosófica de los comportamientos acráticos. Seguidamente mostraré que dicha estrategia no incurre en un RO interteórico.

\subsection{Akrasía y redes neuronales}

La akrasía deriva de una imposición fáctica ligada al refuerzo de redes neuronales. Dependiendo del área funcional a la que esté asociado el refuerzo se puede distinguir diferentes tipos de acción acrática: emocional (por ejemplo, en un sujeto que decide vengarse de otro, a pesar de reconocer que la venganza no es un camino racionalmente preferible), afectiva (en la persona que, aun amando a su pareja, se ve arrastrada a una experiencia de infidelidad) o hedonista (el caso del fumador que sigue fumando a pesar de asumir que sería mejor no hacerlo).

La primera tarea consiste en identificar cuáles son las áreas sinápticas reforzadas que dan pie a los fenómenos acráticos. En los tres tipos que he distinguido (emocional, afectivo o hedonista), la akrasía se produce a raíz de la colisión entre la opción preferida en la deliberación racional y una meta práctica reforzada por redes sinápticas. La pregunta, pues, sería: ¿cómo resulta posible que un proceso mental entre en colisión con otro proceso del mismo rango? La respuesta obvia consiste en que ambos procesos no se hallan en la misma categoría: si así fuera, pasarían a formar parte de una deliberación racional de orden superior (de una metarreflexión). El primer proceso (deliberación racional) forma parte de un subsistema funcional distinto del sistema al que pertenece el segundo (vivencia emocional, afectiva o hedonista reforzada sinápticamente).

Identificar sendos subsistemas será, pues, crucial. Para ello me inspiraré en la distinción tripartita llevada a cabo por Paul D. MacLean, que se complementa 
con los trabajos de Papez (quien puso en relación hipotálamo y corteza límbica) y Yakolev (que mostró el enlace entre hipotálamo, amígdala basolateral y corteza prefrontal orbitaria).

La deliberación racional se halla asociada a la sección del cerebro evolutivamente más reciente, el neocórtex (en expresión de MacLean, «cerebro neomamífero»). Esta afirmación queda confirmada tanto por los estudios en torno al despliegue filogenético de las fases de la hominización como por la aplicación de la imagen de resonancia magnética funcional a los procesos cognitivos. En concreto, parece ser que la corteza prefrontal (por ejemplo, el área 11 de Brodmann) se relaciona particularmente con los procesos deliberativos, aunque en éstos intervengan también otras zonas relacionadas con tareas intelectuales (como las áreas dorsolaterales 9, 10, 45 y 46, las órbito-frontales 12 a 14 y las temporales 10 y 21). En cambio, las vivencias de índole emocional, afectiva o hedonista [a partir de aquí, EAH] se hallan relacionadas con los diferentes núcleos del sistema límbico (amígdala, hipocampo, hipotálamo, núcleo accumbens, giro cingulado, etc.); así pues, conciernen al que MacLean denominó «cerebro paleomamífero ${ }^{4}$.

Los circuitos que conectan ambas áreas son múltiples. Así, por ejemplo, el hipocampo y su conexión con la corteza prefrontal medial a través del circuito de Papez se imbrican en la transmisión de procesos sinápticos relacionados con el mundo EAH, desde los ligados a la emoción y al afecto hasta los relacionados con la gratificación y el placer ${ }^{5}$.

\subsection{El papel del refuerzo neuronal en la akrasía}

El refuerzo de los procesos EAH da lugar a dinámicas mentales de mayor rigidez que las asociadas a la moderación racional; en efecto, dichos procesos poseen un carácter estereotipado característico del cerebro paleomamífero. No es de extrañar que tal refuerzo venga acompañado por una considerable fuerza impositiva, propia de los patrones biopsíquicos a los que se halla ligado filogenéticamente ${ }^{6}$.

¿Cuál es el proceso neuronal que subyace al refuerzo acrático? El mejor candidato es un proceso de facilitación. La facilitación propiamente dicha, es decir, la de índole presináptica, es un fenómeno de este tipo: a raíz de la aplicación

4 Argumentos filogenéticos y observacionales (tanto en experimentos conductuales con animales como por medio de técnicas de observación no invasiva en humanos) apoyan estas afirmaciones. Puede consultarse un elenco de referencias en Moll et al., 2005: 808-809.

5 En otro lugar he tratado la intervención neurocientífica en el sistema límbico y su lectura filosófica al hilo de la estimulación eléctrica del área subcallosa del cingulado (TERUEL, 2013b).

6 En las descripciones desarrolladas en este párrafo hemos de tener en cuenta que cada tarea mental está asociada a la activación de tejidos cerebrales que en la gran mayoría de los casos implican áreas distintas. Tal y como señala José María Delgado, pretender ligar cada tarea a un área específica o a varias áreas perfectamente delimitadas equivale a «balcanizar» la cartografía cerebral (expresión de Joaquín Fuster). 
repetida de un estímulo sobre una sinapsis excitadora, se hace que el potencial de membrana se aproxime al umbral necesario para la apertura de los canales de $\mathrm{Ca} 2+$, con lo que ello implica para la liberación de neurotransmisores y para el origen del potencial de acción entre otros procesos. Ahora bien, los efectos de este proceso son poco duraderos. Cuando se alcanza un cierto umbral de estimulación, en cambio, se produce una potenciación a largo plazo. Los efectos de dicha potenciación son duraderos y, además, específicos: dan lugar a redes definidas de transmisión que mantienen su identidad funcional y que a la vez muestran dinámicas asociativas ${ }^{7}$.

Así pues, la potenciación a largo plazo es un candidato óptimo para el tipo de refuerzo que buscamos. Se da la circunstancia, además, de que se trata de un proceso hondamente enraizado en el sistema límbico y en sus proyecciones a la corteza cerebral. De hecho, el componente cerebral donde mejor ha sido estudiado -más aún: donde comenzó su estudio, de la mano de Lømo y Bliss, en la década de los sesenta en Oslo- es el hipocampo, aunque no se excluye que constituya un fenómeno genérico de la vertiente excitativa del sistema nervioso central. En relación con todo ello se ha conjeturado que ofrezca el modelo cerebral para el aprendizaje, puesto que implica codificación a largo plazo de determinadas pautas sinápticas.

\subsection{Akrasía y $R O$}

Pues bien: a mi modo de ver, esta aproximación a las acciones acráticas reduce intrateóricamente y de modo lícito el planteamiento clásico ligado a la noción de hábito. En efecto, explica cómo es posible que un hábito pueda imponerse no ya en una práctica prerreflexiva sino incluso a la deliberación racional explícita - es decir, resistiéndose al papel rector de la razón deliberativa. Dicho de otro modo: fundamenta el fenómeno de la akrasía. Y lo hace no reduciendo ilícitamente la esfera de lo moral a la de lo neurofisiológico, sino mostrando la conexión entre ambas a través de la modulación llevada a cabo por las redes neuronales. No implica, pues, una tesis materialista (negación de la libertad) sino su contextualización en un cuerpo orgánico (y, por lo tanto, el reconocimiento de su carácter situado).

De este modo, la interpretación neurofilosófica de la akrasía viene a constituir un ejemplo de colaboración entre filosofía y neurociencia que evita el RO interteórico. No se parte aquí de presupuestos ontológica- e interteóricamente reduccionistas (como sí sucede en el marco materialista o en el espiritualista) sino que se intenta perfilar las relaciones biunívocas entre mente y cerebro. El resultado es una comprensión del problema que supera en capacidad explicativa y predictiva a su enfoque clásico. Nuestras acciones influyen en la arquitectura de nuestro cerebro, contribuyen a diseñarla y pueden dar lugar a estereotipos que cristalicen en redes funcionales; podemos decir — desde un

7 En todo ello se debe tener en cuenta el efecto recogido en la ley Weber-Fechner. 
punto de vista neurofisiológico y existencial— que constituyen nuestro destino. Un destino que no está escrito en una impersonal instancia ajena, sino que nosotros contribuimos a forjar.

Sobre la base de lo anterior se puede considerar que el fenómeno de la acción acrática posee carta de ciudadanía en el ámbito de la filosofía de la mente, en el que queda fundamentado tanto desde el punto de vista de los procesos mentales como desde la perspectiva de sus bases neurofisiológicas. Sin restar valor intrínseco a la estructura de la deliberación racional y a sus conexiones con la conciencia moral y la acción, la interpretación filosófica de las aportaciones neurocientíficas permite explicar el fenómeno sobre sólidas bases teóricas y trae consigo consecuencias en el ámbito predictivo. Dichas consecuencias se ponen de manifiesto en su proyección práctica. De ello me ocupo en la tercera sección de este escrito.

\section{APROXIMACIÓN PRÁCTICA AL FENÓMENO DE LA AKRASÍA}

Con «aproximación práctica» me refiero aquí al planteamiento de las acciones acráticas desde el punto de vista de su modificación. El tratamiento psicológico de la akrasía se halla históricamente ligado a tres corrientes. La primera (a la que denominaremos «conductual») se remonta a la teoría aristotélica del hábito; la segunda («genealógica»), a la distinción freudiana relativa a la estructura tripartita del yo; la tercera ( invasiva») hace hincapié en la vertiente farmacológica y quirúrgica. Expondré en primer lugar sus rasgos vertebradores, dejando al margen la enorme variedad que de hecho se da en su interpretación por parte de las diversas corrientes terapéuticas; mostraré entonces a qué se debe la superioridad del enfoque que integra la aportación neurocientífica desde una interpretación ontológicamente no reduccionista. Las siguientes indicaciones no pretenden aportar novedades a la rica e intrincada historia de los modelos de abordaje terapéutico; sí muestran, en cambio, la manera en que de la aproximación neurofilosófica a la akrasía brota un modelo práctico fundamentado in re.

\subsection{Abordaje terapéutico clásico de la akrasía}

\subsubsection{Terapia conductual}

La teoría aristotélica del hábito identifica los patrones de conducta reforzados por repetición como uno de los elementos básicos en la configuración del carácter y por ende de los modelos pedagógicos. Educar el carácter consiste en instaurar hábitos positivos; su instauración lograda equivale a la virtud (hábito operativo en orden al bien). Por tanto, la lucha contra la akrasía consistirá en asentar paulatinamente hábitos que eviten la desviación del resultado deliberativo en que aquélla consiste y que contrarresten, pues, hábitos previos ordenados a una práctica irracional o desdeñable (es decir: vicios). Ahora bien, el uso 
terapéutico del hábito topa con dificultades que provienen de un cierto carácter difuso de éste. Para ilustrar esta afirmación, apliquemos la inversión de hábitos a los ejemplos EAH recurrentes en el presente escrito.

¿Cómo se podría llevar a cabo dicha estrategia en relación con los deseos de venganza? Está claro que deberían ser incoados hábitos contrarios al deseo que se impone en la dinámica acrática. Ahora bien, el espectro de hábitos posibles resulta, cuando menos, difuso: quizá dedicarse a la beneficencia constituya un buen candidato, pero también podría serlo la afición por la pintura, la relajación por medio del yoga o el compromiso político. Del mismo modo, el caso de la persona infiel a su pareja — a la que, sin embargo, ama — admite multitud de proyecciones: en principio, parece que prescindir del refuerzo positivo que implica la presencia del amante constituye la base de cualquier hábito recuperativo; sin embargo, bien puede darse que ello sólo ponga en sordina un deseo que a distancia de tiempo y en la situación adecuada podría emerger sin merma de potencia acrática. Algo similar puede decirse de las conductas que brotan de la imposición de pautas dominadas por sustancias adictivas. En todos estos casos, la modificación de las dinámicas acráticas por medio de la reversión de hábitos resulta ambigua desde el punto de vista procedimental ${ }^{8}$.

8 La narrativa cinematográfica aporta significativos ejemplos de las dinámicas psicológicas a las que me acabo de referir. Así, numerosos films ilustran la dificultad que entraña la lucha contra el deseo (irracional) de venganza. Pensemos, por ejemplo, en Alma, la atribulada enfermera protagonista del film Persona (Ingmar Bergman, 1966). A medida que el desarrollo de su relación con la paciente Elisabeth Vogler le hace percibir lo que ella interpreta como cara oculta de Elisabeth (que incluye, entre otros aspectos, el reflejo de algunos rasgos de su propia personalidad hasta entonces ocultos para ella, como la ausencia de una personalidad consistente), Alma no puede evitar alimentar un odio que se exacerba con rapidez y que se traduce en actos concretos de venganza, aun reconociendo racionalmente que la actitud preferible sería otra (lo cual se muestra en su arrepentimiento). Tanto en este caso como en el de la infidelidad parece que el alejamiento del objeto constituye una condición de posibilidad para la instauración de nuevos modelos de conducta. Así lo entiende la protagonista de Sueños de mujer (Kvinnodröm, Ingmar Bergman, 1955); sin embargo, la distancia geográfica y temporal no consigue eliminar el deseo y la relación prosigue hasta que no se ve truncada por circunstancias externas. Similar estructura se aprecia en Infiel (Trolösa, dirigida por Liv Ullmann sobre guión de Ingmar Bergman, 2000), a pesar del patente rechazo racional de la protagonista y de su profundo arrepentimiento, o en Encuentros privados (Enskilda samtal, Liv Ullmann 1996), aunque en esta última la acción acrática se resuelve con el triunfo de la deliberación racional. El lector apreciará que estos ejemplos remiten al ámbito filmográfico de Ingmar Bergman como director o guionista; a mi modo de ver, se trata de uno de los cineastas que mejor ha reflejado la estructura y consecuencias de la acción acrática. De entre los ejemplos que se puede extraer de su producción, quizá sea De la vida de las marionetas (Aus dem Leben der Marionetten, 1980) el más valioso. El protagonista, Peter Egermann, acude a su psicólogo para manifestarle las pulsiones violentas relacionadas con su esposa Catharina, con la que mantiene una compulsiva relación de amor-odio. La estrategia emprendida por el propio Peter — a saber, buscar satisfacer sus necesidades (sexuales y/o afectivas) a través de su relación con una prostituta - no sólo no elimina el problema sino que lo reprime, dando pie a su emergencia de modo aún más virulento y finalmente trágico. No en vano el film despliega una penetrante reflexión en torno a la libertad, que se halla expuesta de modo perturbador y sucinto en el diálogo del modista Tim con Catharina: 
La estrategia liderada por autores como Albert Ellis puede ser puesta en relación con una vertiente de este modelo. Por su medio se persigue una reestructuración cognitiva que modifique la situación psíquica anómala por medio del esclarecimiento racional de los patrones de apreciación y conducta (cf. por ejemplo Ellis, 1975). Pese a su innegable valor, un planteamiento basado en dicho abordaje racional elude el problema teórico de la akrasía, que por su propia definición esquiva el plano del análisis y la confutación; atribuye, pues, a la deliberación racional una efectividad práctica cuya ausencia o debilidad se halla justamente en la génesis del problema.

\subsubsection{Terapia genealógica}

La distinción freudiana entre la estructura tripartita del yo constituye una segunda fuente relevante en la aproximación psicológica a la akrasía. Como es sabido, en su obra de madurez El yo y el ello (1923) Freud distingue entre el ello (das Es), el yo (das Ich) y el superyó (das Über-Ich). Su teoría sobre la estructura psíquica del ser humano se articula en torno a la tensión entre la instancia pulsional, ligada a los elementos psicosomáticos heredados, propios del fenotipo o reprimidos (Es) — dependientes tanto de la tendencia a la satisfacción del placer (libido) como de la pulsión de muerte-, y la instancia ligada a la interiorización de las normas de comportamiento y del ideal de sí mismo (Über-Ich), dependiente tanto del cumplimiento del deber como del logro de una autoimagen satisfactoria; dicha tensión inconsciente viene parcialmente mediada por la instancia de deliberación racional (Ich) y aflora, en los casos no resueltos, a través de los síntomas neuróticos; éstos no son otra cosa que un desplazamiento somático de tal tensión.

Parece claro que los comportamientos acráticos denotan un desajuste originado por el conflicto entre superyó, yo y ello. Aun cuando el segundo se adhiera a las exigencias presentadas por el primero, es el tercero el que acaba por imponerse. Ahora bien, dicha imposición no se produce sin perjuicio del concepto de sí mismo. La inevitable confusión del sujeto y el deterioro de su identidad consciente constituyen síntomas de lo que Freud denominó "psiconeurosis»" Aunque esta expresión haya caído en desuso en el vocabulario técnico, su sentido se halla fuera de duda: con ella nos referimos a trastornos psicosomáticos

en dicho diálogo, la conciencia del propio proceder acrático se muestra con una viveza inusitada, hasta el punto de que Tim llega a dudar sobre la estructura de su propia identidad - de la que dice sentirse «alejado», como si sus acciones le alienasen de un hipotético núcleo identitario que no coincide con ellas. El problema de la akrasía se manifiesta aquí bajo una luz cruda que evidencia su relevancia antropológica: concierne a la fuente de las acciones y a la configuración de la identidad personal. La confusión del psiquiatra de Peter, expresada en el monólogo conclusivo, muestra a las claras la dificultad de afrontar la modificación del comportamiento acrático.

9 Recogía así el término acuñado por William Cullen en 1769. Se puede rastrear su uso ya en la década de los noventa (Freud, 1898, 19926: 496-497). 
que no poseen un origen geno- o fenotípico sino funcional (radicado, por tanto, en desajustes psíquicos que han sido somatizados ${ }^{10}$.

El tratamiento psicoanalítico de los fenómenos acráticos pasa por su liberación verbal a través de la asociación libre y en un contexto de transferencia. Gracias a ello el paciente se pone en condiciones de descubrir, con la guía del psicoanalista, el origen del que brota la tensión que se manifiesta en la neurosis. Aunque esta regla técnica fundamental haya sido reinterpretada y modificada hasta la saciedad en las escuelas terapéuticas inspiradas por el psicoanálisis, la importancia de la liberación verbal y de la identificación del origen inconsciente del comportamiento constituye una piedra angular incontestada. Llevar a cabo la genealogía del mal implica ya el germen de su sanación, puesto que la tensión oculta deja de serlo y con ello se cercena el desplazamiento somático.

Salta a la vista, sin embargo, que amortiguar la tensión por medio de su desvelamiento puede contribuir al control de los síntomas neuróticos pero no equivale a eliminar la distancia entre yo, ello y superyó; el desajuste entre sus respectivos requerimientos resulta insalvable y ofrece el caldo de cultivo para futuras crisis. Consciente de ello, Freud apunta a la sublimación cultural como liberación del resto libidinal reprimido por la censura de toda laya. La cláusula escéptica con la que concluye El malestar en la cultura (1930) muestra hasta qué punto consideraba problemático el devenir de tal estrategia en un contexto de represión creciente.

\subsubsection{Terapia invasiva}

Con «terapia invasiva» entendemos aquí el abordaje que pretende combatir los comportamientos acráticos por medio de la intervención quirúrgica o del suministro de psicotrópicos. En ambos casos no se negocia con el sujeto acrático —ni por el fomento de hábitos ni a través del diálogo- sino que se interviene en las dinámicas de sus redes neuronales. La historia de dicho tipo de terapias experimenta un considerable auge en la segunda mitad del siglo $\mathrm{xx}$, a raíz de dos fenómenos que constituyen partes integrantes de la tendencia misma. Uno de ellos es el ensayo de intervención quirúrgica en orden a tratar desajustes psíquicos graves, paradigmáticamente representado por la sección del corpus callosum y la lobotomía en casos de pacientes esquizofrénicos (véase los trabajos de Egas Monitz al respecto). El otro es la popularización de los psicotrópicos (psicolépticos, psicoanalépticos, psicodislépticos) para el tratamiento de patologías de creciente presencia como la depresión; su extraordinaria difusión camina paralela a la eficacia en el tratamiento del síntoma y a la borrosidad en la dilucidación de su origen.

Naturalmente, tanto la intervención quirúrgica como el suministro de psicotrópicos tienen su ámbito de justificación. Por otro lado, suscitan lícitos

10 Sobre la cercanía entre la akrasía clásica y la neurosis freudiana se ha pronunciado también G. R. F. FerRARI: "Akrasia as Neurosis in Plato's Protagoras», en Boston Area Colloquium in Ancient Philosophy 6 (1990) 115-150. 
interrogantes sobre su precisión científica y su licitud ética. No es objetivo del presente trabajo abordar estos asuntos, a los que me he aproximado en otro lugar ${ }^{11}$.

La aplicación de psicotrópicos ha sido asociada en ocasiones a los fenómenos acráticos como vía de modificación. Cabe decir, sin embargo, que resulta desproporcionada. Y esto, al menos, por dos motivos. Por un lado, dicha aplicación implica una intervención invasiva - con efectos sobre la disponibilidad de sí mismo del individuo- y ha de ser restringida por ello a casos graves. Por otro, tal estrategia presenta una problemática ambigüedad etiológica y terapéutica. En efecto, el abordaje farmacológico afecta a un vasto espectro de dianas neurofisiológicas y químicas; no existe la posibilidad de intervenir en el preciso punto de conflicto que da lugar al comportamiento acrático. Y esto, a su vez, se debe a dos motivos: el primero es que las redes neuronales configuran un mapa dinámico en el que todo está relacionado con todo; se puede, sí, apaciguar el ánimo de una persona conflictiva, pero esto no sucederá sin afectar a otros rasgos de la personalidad. El segundo motivo es que el comportamiento acrático se halla relacionado con contenidos intencionales: brota de una cierta reacción a vivencias psíquicas (de una injusticia, de un afecto, de un placer, etc.) situadas en un orden no reducible a la mera cartografía electroquímica del cerebro ${ }^{12}$. Todo ello hace que, en línea de principio, la terapia invasiva resulte ambigua en cuanto a sus efectos (y esto, no sólo en el caso del fenómeno acrático $)^{13}$.

\subsubsection{Valoración y crítica de los abordajes precedentes}

Los comportamientos acráticos admiten multitud de aproximaciones. Sin embargo, considero que los procedimientos inspirados en las nociones de vicio y neurosis resultan paradigmáticos por varios motivos. En primer lugar,

11 Lo he hecho, en relación con la terapia basada en estimulación eléctrica del sistema límbico, al hilo de las que he denominado fronteras terapéutica, disciplinar y epistemológica del abordaje invasivo (Teruel, 2013b).

12 Abogar por esa reducibilidad (del orden subjetivo al neurofisiológico) ha sido el error de los psicologismos de toda laya. Las críticas que Edmund Husserl presenta al respecto en sus Investigaciones lógicas resultan suficientemente significativas. En particular, vale la pena llamar la atención sobre las consecuencias y los prejuicios del psicologismo, tal y como aparecen abordados en su primer volumen, «Prolegómenos a la lógica pura» (HusserL, 1900).

13 De nuevo un ejemplo narrativo puede ayudarnos a visualizar este extremo. En el inquietante film Suddenly, last summer (Joseph L. MANKIEwicz, 1959), basado en la obra teatral homónima de Tennessee Williams, la joven protagonista es víctima de un proceso que bien puede calificarse de acrático: parece no poder contener las pulsiones relacionadas con ciertas experiencias del pasado. Por motivos que se revelarán espurios, su tía contrata los servicios de un prestigioso cirujano para practicarle una lobotomía. El problema, tal y como le hace notar el médico, es que junto con los «demonios del alma» quedará sepultada también la personalidad de la joven. En torno a esta terrible efectividad de la intervención quirúrgica, y a la ambigüedad de sus efectos, se desarrolla una trama que llevará a arribar a otro polo terapéutico: el psicoanalítico. 
en ambos casos se trata de patrones explicativos que admiten una traducción práctica con pretensiones predictivas. En segundo lugar, cada uno de ellos abarca un amplio espectro de causas: en el caso del paradigma aristotélico se afronta las pautas reforzadas de comportamiento que oscurecen la deliberación racional; en el paradigma freudiano se aborda el origen inconsciente del conflicto psíquico. Finalmente, cada planteamiento propone un modelo de solución que puede ser reconocido en multitud de enfoques no estrictamente clásicos ni psicoanalíticos: en el primer caso, la terapia conductual o cognitiva asociada al fomento de hábitos teórico-prácticos virtuosos; en el segundo, el análisis ligado al desvelamiento de las tensiones inconscientes. En cambio, el planteamiento invasivo se centra en el combate quirúrgico y farmacológico de los síntomas; de ahí su característica borrosidad etiológica y terapéutica.

Me parece, pues, relevante poner de manifiesto la ambigüedad de dichas estrategias - de suyo, extremadamente valiosas- a la hora de aplicarlas al fenómeno de la akrasía. Podemos preguntarnos si dicha ambigüedad posee una raíz común. A mi modo de ver, la respuesta es afirmativa. A pesar de proporcionar importantes elementos de juicio y tratamiento, ni el abordaje conductual ni el psicoanalítico —ni, aún menos, la terapia invasiva - cuentan con una suficiente aproximación teórica a la estructura psicofísica del fenómeno.

La akrasía halla su origen en el conflicto entre el resultado de la deliberación racional y las pautas de conducta reforzadas neuronalmente. Del planteamiento neurofilosófico se puede extraer, al menos, tres conclusiones: (a) el tratamiento por medio de la incoación de hábitos puede no coincidir con el tipo de abordaje necesario para revertir el refuerzo negativo; (b) desvelar el origen inconsciente de las tensiones que en la akrasía emergen puede contribuir a despojarlas de parte de su componente conflictual, pero no resuelve el problema; y (c) una aproximación práctica adecuada ha de pasar por la desactivación del específico refuerzo sináptico negativo, permitiendo la adhesión a las pautas que brotan de la deliberación racional.

\subsection{Tareas del abordaje neurofilosófico}

Sobre esta base, el tratamiento psicológico de los fenómenos acráticos ha de implicar, a mi juicio, las siguientes tareas:

a) Identificar los procesos mentales que entran en colisión con la esfera deliberativa (sobre la base de lo dicho hasta ahora, procesos de índole EAH);

b) Localizar las redes sinápticas cuyo refuerzo fundamenta el fenómeno (el sistema límbico, con especial atención a proyecciones como los circuitos de Yakolev y de Papez);

c) Diseñar una estrategia de desvinculación de los procesos mentales respecto de las redes sinápticas reforzadas.

d) Implementar, de forma planificada y supervisada, la estrategia de desvinculación. 
De las cuatro fases, la tercera constituye el punto más delicado. En línea de principio, las sinapsis reforzadas asociadas a procesos EAH pueden venir desvinculadas de los procesos mentales considerados desdeñables frente a otros preferibles; dicha desvinculación puede acaecer por cauce negativo o positivo. En el primer caso (desvinculación negativa), se trataría de disociar el proceso mental en cuestión de su carácter reforzado; puesto que el objeto intencional de dicho proceso se halla biológica y psicológicamente ligado a respuestas de tipo EAH, tal disociación resulta artificial y sólo podría ser producida a través de estimulaciones biofísicas repetidas - por ejemplo, por medio de su asociación pavloviana con estímulos perjudiciales (método rechazable por sus implicaciones deshumanizadoras) o del suministro de psicotrópicos. La vía de desvinculación positiva resulta más adecuada: no sólo no actúa de modo traumático sino que no consiste en negar la dimensión EAH de los procesos mentales racionalmente desdeñables; ésta ha de ser reorientada, cosa que puede suceder por medio de su vinculación a asociaciones preferibles y que precisa de un marco narrativo, hermenéutico: un marco — tal y como autores como Viktor Frankl han apreciado con acierto- asociado a un horizonte de sentido (cf. por ejemplo Frankl, 1946).

La tercera variante (hedonista) de la akrasía presenta, por su carácter básico y estereotipado, el caso menos complejo. Pensemos en el fumador que sigue consumiendo tabaco a pesar de que es consciente de que sería preferible no hacerlo. Los elementos que intervienen en el fenómeno son relativamente pocos: la práctica del consumo, el efecto narcotizante y la repetición de la práctica con el consiguiente refuerzo de las sinapsis asociadas al placer (al que se pueden unir efectos pavlovianos). La desvinculación positiva habrá de pasar por la reorientación de los elementos recuperables del fenómeno: así, la estimulación placentera habrá de ser paulatinamente sustituida por otra fuente; sólo cuando la desconexión se halle mínimamente garantizada se podrá proceder a eliminar la fuente sustitutoria de estimulación. La práctica terapéutica de los últimos años, en conexión con la investigación neurocientífica, ha mostrado la viabilidad del tratamiento por desvinculación positiva en la variante hedonista de la akrasía: lo demuestra el éxito creciente de las terapias encaminadas al abandono del tabaquismo. Dicho resultado era de esperar, teniendo en cuenta el carácter estructuralmente menos complejo de estos comportamientos acráticos.

Más difícil parece el abordaje terapéutico de la akrasía en sus variantes emocional y afectiva, puesto que en ambas se halla implicada la forja de conceptos abstractos (pensemos, por ejemplo, en la noción de justicia y su asociación a la venganza) o de parámetros afectivos (como en el caso de la infidelidad); estas variantes incluyen una mayor profusión de procesos mentales, con bases neurofisiológicas muy diversificadas. De ahí que el tratamiento resulte arduo y no admita recursos farmacológicos como los empleados en la variante hedonista. Sin embargo, esto no excluye su abordaje. La vía de la desvinculación positiva sigue resultando adecuada, en la medida en que la desconexión del comportamiento racionalmente desdeñable constituye un objeto cuyo logro resulta, en principio, viable (según grados de dificultad que dependen tanto de 
los factores en juego como del grado de refuerzo de las redes sinápticas ya implicadas). Condición para ello es que las necesidades EAH a las que responde dicho comportamiento vengan a ser cubiertas desde un horizonte de sentido racionalmente preferible. Diseñar la estrategia oportuna requerirá una estrecha colaboración entre las personas involucradas en el proceso, de modo que el individuo pueda reconquistar un contexto en el que la deliberación racional ocupe de nuevo el lugar que le corresponde en la vida mental y en su reflejo práctico.

Facilitar aquí ulteriores detalles precisaría una prolija casuística. Excede, pues, el sentido de esta sección, cuyo objetivo era mostrar el modo en que el abordaje neurofilosófico puede complementar los modelos de tratamiento de los fenómenos acráticos.

\section{REFLEXIONES CONCLUSIVAS}

Pablo de Tarso ha acuñado una de las expresiones más tajantes de la dicotomía que el comportamiento acrático trae consigo. Lo hizo en su carta a los habitantes de Roma: «Porque lo que hago, no lo entiendo; pues no hago lo que quiero, sino lo que aborrezco, eso hago» (Rom 7, 15); y más adelante: «Porque no hago el bien que quiero, sino el mal que no quiero, eso hago» (Rom 7, 19). Pocas décadas atrás, Ovidio había acuñado al respecto una expresión diamantina: «Video meliora proboque, deteriora sequor», por cuyo medio Medea reconocía inclinarse hacia lo peor aun viendo lo mejor y aprobándolo (Metamorfosis VII, 20-21). Nosotros hemos querido aproximarnos al fenómeno de la akrasía desde un punto de vista neurofilosófico, a saber, a partir de la interpretación filosófica de las bases y dinámicas neurofisiológicas de la toma de decisiones.

El primer punto de nuestra argumentación (relativo a la comprensión teorética de la akrasía) nos ha permitido afrontar un problema clásico con herramientas procedentes de un RM moderno, a saber: las del análisis neurocientífico de las bases neurofisiológicas relacionadas con el conflicto acrático. La interpretación filosófica de dicho análisis ha evitado la caída en un (ilícito) RO interteórico que hubiera traído consigo la negación del papel que la deliberación racional juega en el proceso - y, con ella, la desaparición de la conciencia moral y la libertad del horizonte del problema. Nuestro planteamiento toma nota de la intervención de la deliberación racional y de su suplantación práctica (acrática), para resolver ésta recurriendo a la identificación de las estructuras y dinámicas sinápticas implicadas en sendos procesos.

La argumentación desplegada en el último punto (relativo a la praxis terapéutica) nos ha servido para ilustrar nuestra comprensión de la akrasía. Se trata de desarrollos que encuentran eco en los modelos psicoterapéuticos de las últimas décadas - en particular, en los que surgen de la psicología humanista en el siglo xx (pensemos en las aportaciones de autores como Erich Fromm, Karl Jaspers o el ya citado Viktor Frankl, entre otros). Notemos, por ejemplo, 
que su énfasis en la potencial positividad de las dinámicas biofísicas y en la deseable subordinación de éstas a la autorrealización global de la persona entronca coherentemente con nuestra valoración de los elementos EAH de los fenómenos acráticos y con nuestra insistencia en la desvinculación positiva como estrategia válida para ponerlos al servicio de la persona. De esta manera, nuestra aproximación se propone refinar el abordaje asociado a la noción de hábito y superar — desde el preciso punto de vista que hemos adoptado- tanto las ambigüedades de la genealogía psicoanalítica como las imprecisiones de la terapia psicotrópica, reconociendo a la vez sus valiosas e insoslayables aportaciones a una teoría y praxis en torno a la psique humana.

Por otro lado, el modelo de intelección de la akrasía aquí expuesto constituye un ejemplo de interpretación filosófica de los datos neurocientíficos que conjuga el RM con su inclusión en un modelo antropológico no reduccionista; conjura, pues, el riesgo de RO interteórico. A mi juicio, la comprensión de la investigación neurocientífica encontrará su vía auténticamente contemporánea en la medida en que rehúya la pendiente fácil y poco satisfactoria del materialismo (clásico, emergentista o eliminativista) y se vincule a una antropología ontológicamente no reduccionista. El ejercicio llevado a cabo en estas páginas puede ofrecer una pequeña aportación en este sentido.

\section{REFERENCIAS BIBLIOGRÁFICAS}

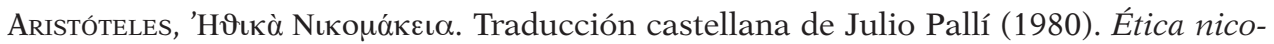
máquea. Madrid: Gredos, 2011.

Davidson, D. $\left(1969^{1}\right)$, «How is Weakness of the Will Possible?». En Essays on Actions and Events. Oxford: Oxford University Press, 21-42.

Delgado, J. M. (2011), «Decidir no es cosa de dos (Un ensayo sobre la neurofisiología de la toma de decisiones)». Revista de Occidente 356, 19-39.

Ellis. A. (1957), How to Live with a Neurotic. Oxford: Crown Publishers.

Ferrari, G. R. F. (1990), «Akrasia as Neurosis in Plato's Protagoras». Boston Area Colloquium in Ancient Philosophy 6, 115-150.

FrankL, V. (1946), Ärztliche Seelsorge. Grundlagen der Logotherapie und Existenzanalyse. Viena: Deuticke.

Freud, S. (1923), Das Ich und das Es. Leipzig / Viena / Zúrich: Internationaler Psychoanalytischer Verlag.

- (1898), «Die Sexualität in der Ätiologie der Neurosen». Wiener Klinische Rundschau 2, 4, 5, 7. En Gesammelte Werke, vol. I (19926), Fráncfort del Meno: Fischer Verlag.

Fuster, J. M. (2009), The Prefrontal Cortex. Ámsterdam: Elsevier.

Moll, J., Zahn, R., De Oliveira-Souza, R., Krueger, F., Grafman, J. (2005), «The neuronal basis of human cognition». Nature Reviews. Neuroscience 6, 799-809.

Holton, R. (1999), «Intention and Weakness of Will». The Journal of Philosophy 96, 5, 241-262.

Husserl, E. (1900), Logische Untersuchungen. Halle an der Saale: Max Niemeyer.

Joyce, R. (1995), «Early Stoicism and Akrasia». Phronesis: A Journal for Ancient Philosophy 40, 3, 315-335. 
MacLean, P. D. (1989), The Triune Brain in Evolution: Role in Paleocerebral Functions. Nueva York: Plenum Press.

- (1975), «Sensory and perceptive factors in emotional functions of the triune brain». En LÉvy, L. (ed.), Emotions. Their Parameters and Measurement. Nueva York: Raven Press, p. 71-92.

Mele, A. R. (1992), «Akrasia, Self-Control, and Second-Order Desires». Noûs 26, 3, 281 302.

- (1989), «Akratic Feelings». Philosophy and Phenomenological Research 50, 2, 277 288.

- (1986), «Is Akratic Action Unfree?». Philosophy and Phenomenological Research 46, 4, 673-679.

SANTAS, G. (1969), "Aristotle on Practical Inference, the Explanation of Action, and Akrasia». Phronesis 14, 162-189.

Teruel, P. J. (2013a), «La tensión oculta en la metapsicología freudiana como índice del debate contemporáneo entre los paradigmas humanista y naturalista». Cuadernos salmantinos de filosofía 40, 379-392.

- (2013b), «La encrucijada neurocientífica entre naturalismo y humanismo. Análisis filosófico de algunos tratamientos psiquiátricos por estimulación eléctrica del sistema límbico». Daímon. Revista Internacional de Filosofía 59, 103-113.

- (2013c), «El doble sentido del reduccionismo científico. De Galileo, Darwin y Freud al monismo irrestricto: análisis crítico y propuesta del «único monismo posible». Naturaleza y libertad 2, 191-222.

Universitat de València

Pedro Jesús Teruel

pedro.teruel@uv.es

[Artículo aprobado para publicación en octubre de 2016] 